\title{
The Fourier-Kelvin Stellar Interferometer (FKSI): a progress report and preliminary results from our nulling testbed
}

R. K. Barry, W. C. Danchi, V. J. Chambers, J. Rajagopal, L. J. Richardson, et al. 


\title{
The Fourier-Kelvin Stellar Interferometer (FKSI): A progress report and preliminary results from our nulling testbed
}

\author{
R. K. Barry ${ }^{\mathrm{a}, \mathrm{i}}$, W. C. Danchi ${ }^{\mathrm{a}}$, V. J. Chambers ${ }^{\mathrm{a}}$, J. Rajagopal ${ }^{\mathrm{a}, \mathrm{g}}$, L. J. Richardson ${ }^{\mathrm{a}}$, A.

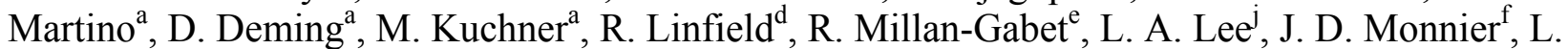 \\ G. Mundy ${ }^{\mathrm{g}}$, C. Noecker ${ }^{\mathrm{d}}$, S. Seager ${ }^{\mathrm{h}}$, D. J. Wallace ${ }^{\mathrm{a}}$, R. J. Allen ${ }^{\mathrm{b}}$, W. A. Traub ${ }^{\mathrm{c}}$, H. C. Ford ${ }^{\mathrm{i}}$. \\ ${ }^{a}$ NASA Goddard Space Flight Center, Greenbelt, MD 20771, USA \\ ${ }^{\mathrm{b}}$ Space Telescope Science Institute,3700 San Martin Dr., Baltimore, MD 21218, USA \\ ${ }^{c}$ Harvard-Smithsonian Center for Astrophysics, 60 Garden St., Cambridge, MA 02138, USA \\ ${ }^{\mathrm{d}}$ Ball Aerospace, Boulder, 1600 Commerce St., CO 80301, USA \\ ${ }^{\text {e}}$ California Institute of Technology, 1201 E. California Blvd., Pasadena, CA 91125, USA \\ ${ }^{\mathrm{f}}$ University of Michigan, Astronomy Department, Ann Arbor, MI 48109, USA \\ ${ }^{\mathrm{g}}$ University of Maryland, Astronomy Department, College Park, MD 20742, USA \\ ${ }^{\mathrm{h}}$ Carnegie Institution of Washington, 5241 Broad Branch Rd., NW, Washington, DC 20015, USA \\ ${ }^{\mathrm{i}}$ The Johns Hopkins University, 3400 N. Charles St., Baltimore, MD 21218 \\ ${ }^{\mathrm{j}}$ Princeton University, Princeton, New Jersey 08544 USA
}

Keywords: nulling interferometer, symmetric Mach-Zehnder nuller, extrasolar planets, giant planets, planet formation, debris disk, habitable zone

\begin{abstract}
The Fourier-Kelvin Stellar Interferometer (FKSI) is a mission concept for an imaging and nulling interferometer for the near infrared to mid-infrared spectral region (3-8 microns). FKSI is a scientific and technological pathfinder to TPF/DARWIN as well as SPIRIT, SPECS, and SAFIR. It will also be a high angular resolution system complementary to JWST. There are four key scientific issues the FKSI mission is designed to address. First, we plan to characterize the atmospheres of the known extra-solar giant planets. Second, we will explore the morphology of debris disks to look for resonant structures to find and characterize extrasolar planets. Third, we will observe young stellar systems to understand their evolution and planet forming potential, and study circumstellar material around a variety of stellar types to better understand their evolutionary state. Finally, we plan to measure detailed structures inside active galactic nuclei. We report results of simulation studies of the imaging capabilities of the FKSI with various configurations of two to five telescopes including the effects of thermal noise and local and exozodiacal dust emission. We also report preliminary results from our symmetric Mach-Zehnder nulling testbed.
\end{abstract}

\section{SCIENTIFIC OBJECTIVES OF THE FKSI MISSION}

The high spatial and spectroscopic resolving capability of the FKSI instrument together with its calculated sensitivity will position it as an important facility for the study of a range of astronomical phenomena. Its science objectives are, very broadly, to directly detect extrasolar giant planets (EGP), study debris disks and the evolution of protostellar and evolved stellar systems, and to facilitate the study of extra-galactic star formation regions and the extended neighborhoods of active galactic nuclei.

The detection and study of EGPs will be greatly facilitated by a dedicated interferometer on a satellite platform operating at infrared wavelengths [1]. To answer key questions about these planets FKSI will be able, depending on configuration, to detect at least 25 known EGPs and make precise determination of their orbital parameters [2]. (See Fig. 1.) These parameters may then be used to constrain orbit models of the detected system and to allow calculation of orbital inclination. Historically, only the star's reflex motion (due to the presence of one or more planets) along our line

Techniques and Instrumentation for Detection of Exoplanets II, edited by Daniel R. Coulter, Proceedings of SPIE Vol. 5905 (SPIE, Bellingham, WA, 2005) $\cdot 0277-786 \mathrm{X} / 05 / \$ 15 \cdot$ doi: 10.1117/12.627827 
of sight, i.e., radial velocity, has been successfully measured. Determination of radial velocity determines only the range of planet masses possible given the stellar mass. Using the laws of motion and simple trigonometry it is easily shown that the radial velocity data allows determination of the mass times the sine of the orbital inclination, (i), rather than the mass itself. This results in the so-called $\sin (i)$ ambiguity of one of the planet's principle characteristics. Measurements conducted using FKSI will allow resolution of this ambiguity, through determination of orbital inclination and will enable calculation of the object's true mass. Under favorable eclipsing circumstances, data from FKSI may further allow inference of the planet's bulk density - the parent star's diameter may be estimated from its spectral class and the amount of dimming would be proportional to the planet's radius.

The spatial resolution of the Fourier-Kelvin Stellar Interferometer together with the instrument's $\lambda \Delta \lambda \sim 25$ spectroscopic capability will allow the direct detection and analysis of photons from EGP under certain conditions of orbital phase and angular separation. These measurements will facilitate studies of the environmental conditions to which the planet is subject. They will also provide a means of measuring the composition of the planet's atmosphere. Taken together, these data will allow scientists to gain insight into the evolutionary history of the planetary system - a key scientific goal. The development of such techniques by the community involved in the study of EGPs may then be adapted for use by TPF scientists in the characterization of Earth-like planets around distant stars.

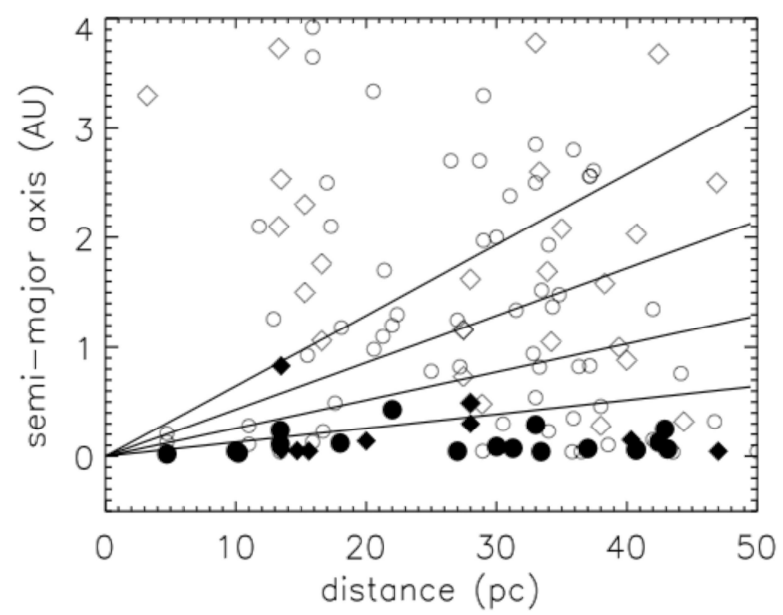

Fig. 1. Distance (in parsecs) and orbital radius (semi-major axes, in AU) for 140 of the known extrasolar planets. The planets within at least a \pm 40 degree field of regard, and nominally detectable by FKSI, are plotted with filled symbols. Circles indicate planets within a 20 degree field of regard, and diamonds indicate additional detectable planets if the field of regard is extended to \pm 40 degrees. The lines correspond to the first fringe maximum for nulling interferometers of baselines 8, 12, 20, and $40 \mathrm{~m}$ (top to bottom).

While direct detection of EGPs is a principle goal of the FKSI mission, study of stellar environments in general will be greatly facilitated by the instruments spatial and spectral resolving power as well. With FKSI, scientists may observe the formative stages of planetary systems, protoplanetary disk structure, the evolution of primordial exozodiacal dust, and debris disks. These observations may then be used to determine the characteristics of circumstellar material that could lead to planet formation thereby refining the target lists and science goals of the TPF mission.

Another scientific goal of FKSI is the study of the environment of nearby sub-giant (luminosity class IV) and giant (luminosity class III) stars. It seems reasonable to hypothesize that these evolved objects could have planetary systems with the same frequency as zero-age main sequence stars (luminosity class V) for a given metallicity. Furthermore, the habitable zones of such systems, conservatively defined as a shell region around a star where planetary surface liquid water could be found, have been shown by Lopez, Schneider, and Danchi [3] to slowly grow in width and radius as the star evolves off of the main sequence. This departure from the main sequence, which begins by a movement of the star along the sub-giant branch of the Hertzsprung-Russel diagram, continues through an ascent up the red giant branch and ends as an extended core helium burning phase, can take up to several $10^{9}$ years depending on the initial mass of the star 
and other parameters such as metallicity. The authors further suggest that life may exist on a planet located in a range of from 2-22 AU when both the first stage of post-main-sequence stellar evolution and post-helium-flash epochs are included depending on how long it takes for life to first appear. Importantly, if planets could be detected within the habitable zones of evolved stars and if these planets showed evidence of life such as atmospheric biomarkers, it would then be possible to determine an empirical lower limit of the timescale for the formation of life. Determination of this timescale from evidence gathered on Earth has always been complicated by the fact that life first appeared while the Earth was undergoing a period of heavy meteoric bombardment. Some of these collisions were almost certainly energetic enough to completely sterilize the planet and would have repeatedly restarted the clock on evolution. As a result the fossil evidence for this early period is somewhat ambiguous.

A last important science driver for FKSI would be the empirical determination of characteristics of pre and post-mainsequence stars through careful observation of binary stellar pairs in which one companion is such an object. These observations could then be used to constrain astrophysical models of stellar evolution. Such observations would be greatly facilitated through the use of a seismically isolated, thermally stable, high spatial resolution, on-orbit instrument operated as a Bracewell interferometer.

\section{FKSI MISSION CONCEPT AND DESIGN APPROACH}

Our team, consisting of scientists from a broad array of institutions together with engineering support from GSFC, has expended significant effort to develop a range of design options for FKSI. We have studied various beam combination techniques and array architectures in preparation for submission of FKSI as a Discovery-class mission. These design studies were conducted initially at GSFC's Instrument Synthesis and Analysis Laboratory and the Integrated Mission Design Center - important functions within GSFC's infrastructure used to facilitate rapid vetting of various mission and instrument design concepts. These studies were then augmented by the work of a larger, focused team of experienced scientists and engineers dedicated to the FKSI mission. The resulting design is a nulling interferometer configuration with an optical system consisting of two $0.5 \mathrm{~m}$ telescopes on a $12.5 \mathrm{~m}$ boom feeding a symmetric Mach-Zehnder beam combiner [4]. A null tracker and hollow-core fiber for wavefront cleanup further augment the system and allow it to produce the required $10^{4}$ null of the central starlight. Fig. 2 shows the requirements derivation from the mission's science goals and the flowdown to key technologies. A thorough description of the design study and technology trade process was published recently [5]. Discussion of the complete error budget for this design including the above trades is beyond the scope of this article and is presented in papers by Hyde et al $[6,7]$.

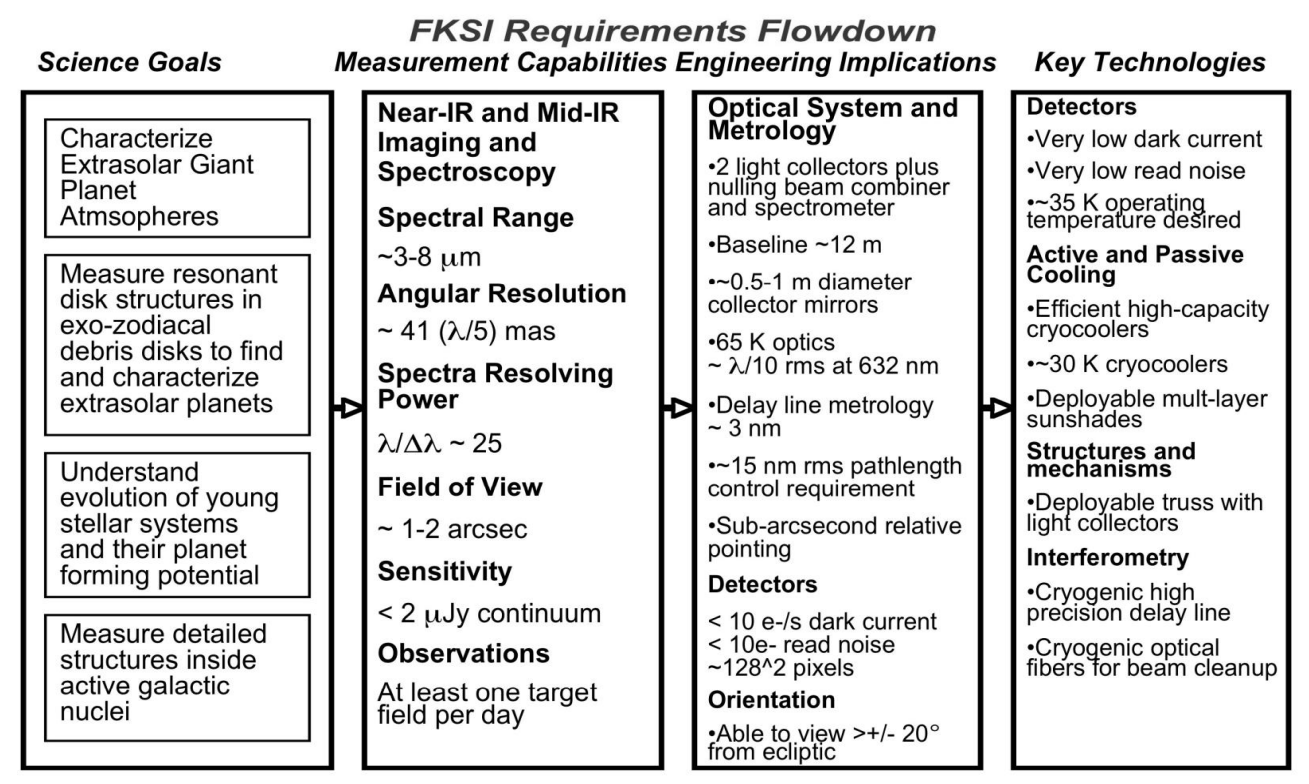

Fig. 2. The FKSI requirements flowdown. 
Fig. 3 shows the design realization of the Fourier-Kelvin Stellar Interferometer as outlined above. The FKSI testbed, described in some detail below, consists primarily of components in the nulling instrument assembly block.

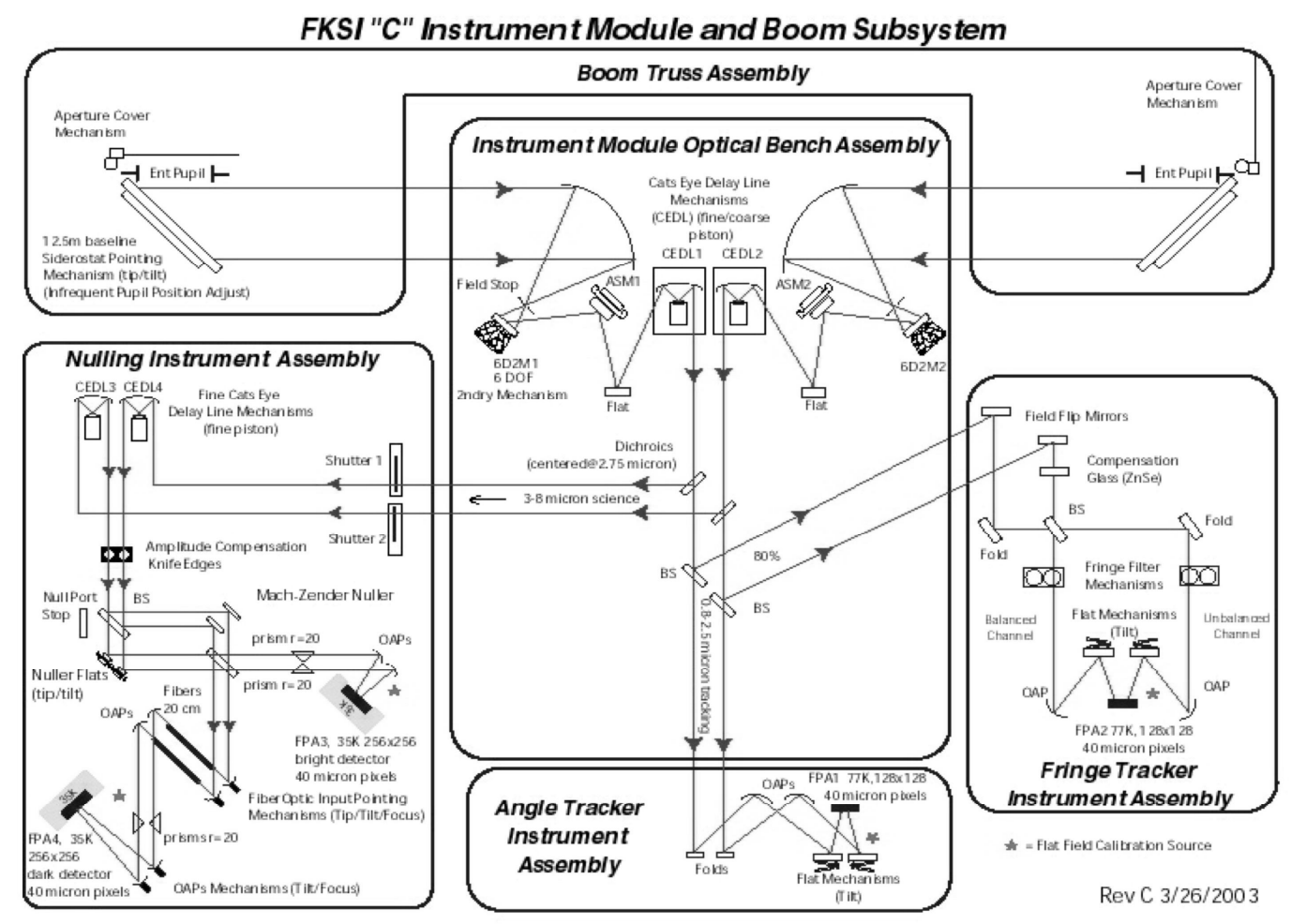

Fig. 3. The FKSI instrument module and boom subsystems.

\section{THE FKSI NULLING TESTBED}

The FKSI testbed is now fully funded and is being built in the Horizontal Flow Facility at NASA's Goddard Space Flight Center in Greenbelt, Maryland. This testbed will allow the instrument designers to evaluate technically challenging aspects of the design. In particular, the instrument's symmetric Mach-Zehnder nulling architecture will be examined together with a novel ditherless quadrature phase detector approach. Significant progress has already been made on the testbed, which is in transition from visible to monochromatic IR testing.

The nulling architecture of the testbed consists of a symmetric Mach-Zehnder beam combiner. A laser beam is collimated by an off-axis parabola, which is then passed through a mask with two apertures. This mask simulates the beams coming from the siderostats of the proposed two-aperture version of the FKSI design. These beams then enter a pair of mirror-symmetric right angle periscopes, and are rotated 180 degrees resulting in a field reversal, which affects the incident polarization states by reversing the $s$ and $p$ plane reflections. The beams are then relayed through cat's-eye retro-reflectors in order to control the interference of the beams in delay space without effecting polarization. Although the monochromatic sources make the testbed less sensitive to dissimilar path lengths because of their great coherence length, the ability to equalize path lengths will be crucial when a white light source is used. After the beams are passed through the delay line, two beamsplitters split both beams, and two more beamsplitters re-combine them. The beamsplitters and the fast steering mirrors are controlled by picomotors for fine adjusting. All four recombined beams are sent to four detector channels, which are used to gather both scientific data and metrology.

The build-up of the FKSI testbed began with locating laboratory space in NASA, Goddard Space Flight Center's Horizontal Flow Facility on a Newport $182 \mathrm{~cm} \times 122 \mathrm{~cm}$ isolation table. A ray trace design was created to fit the 
breadboard table with $\mathrm{X}, \mathrm{Y}$ and $\mathrm{Z}$ coordinates for each optic as well as surface rotations Rx, Ry and Rz. (See Fig. 4.) We printed this design directly onto the table surface placing the ray-trace coordinate origin just before the field-split mirror. All folds, beamsplitters and periscopes were mounted roughly $(+/-\sim 10 \mathrm{~mm})$ to resolve possible space-constraint issues with the hardware. The fold and parabola of each cat's-eye retro-reflector were aligned in $\mathrm{Rx}$ and Ry simultaneously with $\mathrm{Z}$ by auto-collimating with a T3000 theodolite while placing each optic in $\mathrm{Z}$ with a cathetometer. The laser source is currently collimated off a section of a parabola before being folded onto the field split mirror.

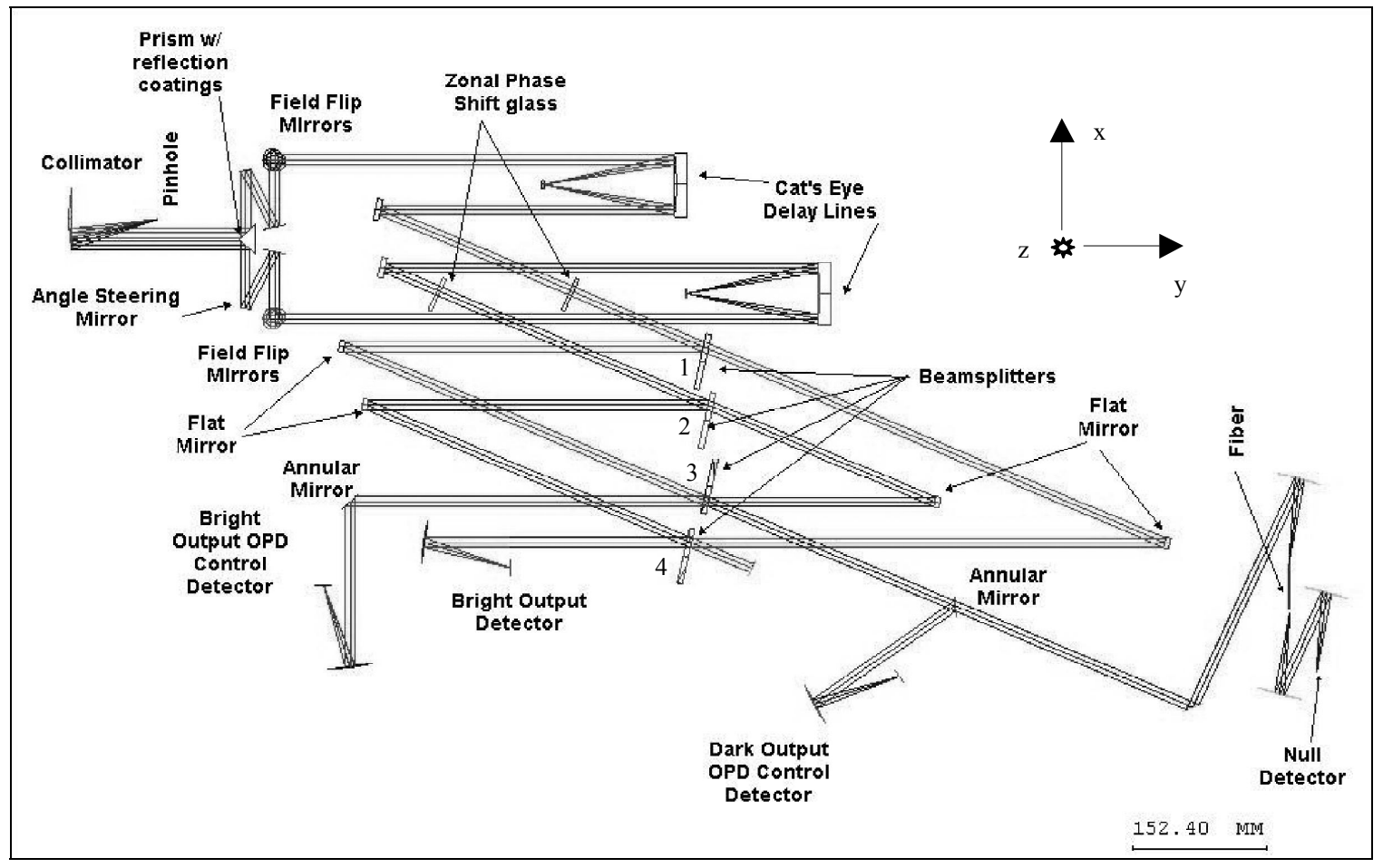

Fig. 4. The FKSI nulling testbed optical layout.

In order to more accurately place each optic in $\mathrm{X}, \mathrm{Y}$ and $\mathrm{Z}$, a 2 × 9mm aperture mask with cross-hairs was mounted before the field-split mirror, to simulate the two beams entering the interferometer from the instrument booms. The crosshair images were used to place all optics to within $\sim 2 \mathrm{~mm}$, allowing the first fringes to be produced on the testbed. Optics were further adjusted in Rx and Ry using theodolite triangulation. After the optics were re-adjusted, we mounted two additional iris-masks to mark the position of the aligned beams after the beamsplitters and removed the front aperture mask. Removing the front mask cleaned the wavefront of diffraction rings and allowed clearer, more visible fringes. We placed CCD cameras in three of the outputs and further adjusted beamsplitters to null the four channels simultaneously. A detector was mounted in the remaining beam (science channel) and fringe contrast measurements were taken by dithering one arm of the interferometer.

Several measurements were taken to better align the interferometer for improved fringe contrast. With the visible light, commercial, off-the-shelf (COTS) optics and light source, there was an unavoidable degree of amplitude mismatch for each of the four beams. Optical power throughput as a function of angle (Rx) was measured for several optics and power measurements were taken before the split mirror to adjust the front mask placement. Amplitude mismatch was further addressed by taking measurements of $\mathrm{P}$ and $\mathrm{S}$ polarization rotation after each optic to match up transmitted and reflected polarization fields in the detector space. The degree of rotation was smaller than the margin or error for our measurements.

In order to assure that we have identified the sources of contrast reduction in our current visible light system and in preparation to move to an infrared source, we derived a simple mathematical model of instrument throughput. Our 
results employ the use of Eqn. 1 given below where $\Omega$ represents a visibility amplitude factor, $\Phi$ is the relative pathlength difference as a fractional part of a wave $(\Phi=2 \pi(\Delta \mathrm{L}) / \lambda)$ and $\Delta \mathrm{L}$ is the path length difference in meters. $\mathrm{I}_{\mathrm{T}}$ is the optical power of the transmitted beam through the optical combiner and $I_{R}$ is that of the reflected beam. Since both $I_{T}$ and $I_{R}$ are composed of both $S$ and P polarized light, we expanded the equation and also considered whether each DC portion $\left(\mathrm{I}_{\mathrm{T}} \mathrm{s}, \mathrm{I}_{\mathrm{R}} \mathrm{p}\right.$, etc.) is composed of a combination of the $\mathrm{S}$ and $\mathrm{P}$ fields. Since laboratory measurements show changes in polarization angle, $\theta$, are nearly zero as the beams go through the system, components $I_{x} s$ or $I_{x} p$ reduce to $I_{T}$ and allow us to use the simplified Eqn. 3 for calculating fringe visibility. The function describing optical power is

$$
I=I_{T}+I_{R}+2 \Omega \sqrt{I_{T} I_{R}} \operatorname{Cos} \Phi
$$

where

and

$$
I_{T}=I_{T s}+I_{T p}
$$

$$
I_{R}=I_{R s}+I_{R p}
$$

So that, by substitution,

$$
I=\left\{I_{T s}+I_{T p}+I_{R s}+I_{R p}\right\}_{D C}+2 \Omega\left[\sqrt{I_{T s} I_{R s}}+\sqrt{I_{T p} I_{R p}}\right] \operatorname{Cos} \Phi .
$$

This expression reaches its extrema at the maximum and minimum of the cosine function, and, when substituted in to the equation for visibility,

$$
F=\frac{I_{\max }-I_{\min }}{I_{\max }+I_{\min }}
$$

reduces to the amplitude term multiplying the cosine function in Eqn. 1 divided by the DC term which is the mean value.

$$
F_{r}=(2 \Omega)\left[\sqrt{I_{T_{S} I_{R s}}}+\sqrt{I_{T_{p} I_{R p}}}\right] /\left[I_{T_{s}}+I_{R s}+I_{T_{p}}+I_{T_{p}}\right]
$$

By using an aperture stop and measuring the increase in fringe contrast as we reduce the stop size, we determined that the reduction in visibility from wavefront errors was $\sim 0.4 \%$. Separated S and P polarized light measurements result in fringe contrast values of $98.51 \%$ and $98.49 \%$. Results from treating S and P polarizations as one come close to their average at $98.5 \%$. We conclude that polarization mismatch is negligible from this measurement. When we account for the wavefront error contribution to $\Omega$ and use directly measured optical power in Eqn. (3), there is a consistent $10 \%$ difference between calculated contrast and measured contrast. We hypothesized that this discrepancy could be due to ghost beam reflections inside the beamsplitters and conducted calculations, given below, to determine if this idea had merit.

By ignoring polarization effects, we may calculate the effect of ghosting on the fringe visibility to see if its contribution could explain the 10\% discrepancy between our calculated and measured fringe contrast. The fringe visibility Eqn. 3 may be modified to include a ghost image power term in Eqn. 4. This simplified equation for fringe visibility shows more directly how ghost beams reduce the fringe quality.

$$
F_{r}=(2 \Omega)\left\lfloor\sqrt{I_{T s} I_{R s}}+\sqrt{I_{T_{p}} I_{R p}}\right\rfloor /\left[I_{G}+\left(I_{T_{s}}+I_{R s}+I_{T_{p}}+I_{R p}\right)\right]
$$


Measuring the optical power throughput of each beamsplitter and finding the transmittance of the anti-reflection coatings allows us to determine the power of each ghost beam. The COTS Melles Griot beam splitters' HEBAR® antireflection coatings exhibit a transmittance of $87 \%$ at $12^{\circ}$ incidence. Their transmittance would be $98-99 \%$ if set at the optimum $45^{\circ}$. In the case of beamsplitter 1 , the incident power is $136 \mu \mathrm{W}$, the total reflected power is $18.8 \mu \mathrm{W}(14.3 \%)$ and the total transmitted is $113 \mu \mathrm{W}(85.7 \%)$. Fig. 5 shows the reflected and transmitted beams of beamsplitter 1 as well as the $1^{\text {st }}$ order ghost beams. Both ' $r$ ' and ' $t$ ' are known quantities. ' $R$ ' and ' $T$ ' must be calculated by setting the transmitted and reflected beam components equal to their measured power percentages. As such: $\mathrm{R}+\mathrm{Tr}+\mathrm{C}=14.34 \%$ and $\mathrm{Tt}+\mathrm{TrRt}+\mathrm{C}=85.7 \%$. $\mathrm{C}$ is the remaining percentage due to $2^{\text {nd }}$ and higher order ghost beams and is equal $0.015 \%$ - an insignificant contribution. Solving for $\mathrm{R}$ and $\mathrm{T}$ separately gives $\mathrm{R}=0.14-0.13 \mathrm{~T}^{2}$ (series 2$)$ and $\mathrm{T}=0.86 /(0.11 \mathrm{R}+$ 0.87 ) (series 3). Solving simultaneously gives $-0.01 \mathrm{~T}^{3}+0.89 \mathrm{~T}-0.86=0$ (series 1 ). These yield a transmittance $(\mathrm{T})$ of $98.2 \%$. The ghost beam component from beamsplitter 1 that strikes our detector is labeled as TrRt and is equal to $0.982(0.13)(0.018)(0.87)=0.2 \%$. Compared to the $1^{\text {st }}$ order transmitted percentage of $85.4 \%$, the ghosting percentage from beamsplitter 1 is small, yet roughly one third strong enough to cause the identified $10 \%$ drop in fringe visibility.
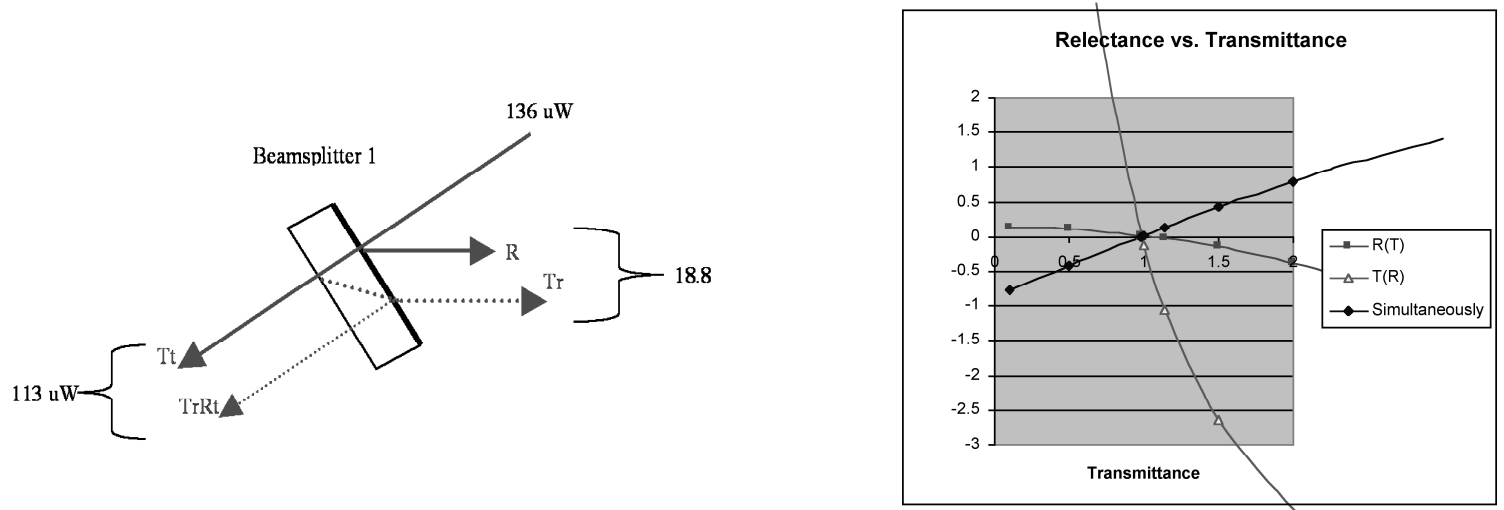

Fig. 5. Ghost image calculation and beam splitter graph.

The ghost beam created by the nominal beam reflecting off beamsplitter 4 contains a much greater share of the power throughput. A full $10.3 \%$ of $21.3 \%$ reflected power contributes to a total of $55.2 \mu \mathrm{W}$ of ghost power for this beam path alone. The other interferometer arm contributes a total of $3.2 \mu \mathrm{W}$ of ghost power from beamsplitters 2 and 4 . Entering a combined ghost power component of $58.4 \mu \mathrm{W}$ in Eqn. 4 as a DC term yields a very low fringe visibility of $33 \%$. Reexamining the power percentages from beamsplitter 4 shows that the $55.2 \mu \mathrm{W}$ of reflected ghost power is nearly equal to the $56.8 \mu \mathrm{W}$ of power in the nominal reflected beam. The angle of the beamsplitter has created a ghost beam component as strong as the nominal beam. In this situation, it is likely that the ghost beams themselves are producing fringe power in addition to contributing to the DC components of the beam intensities. From these results it is reasonable to assume that such a substantial degree of ghosting could create the $10 \%$ fringe contrast shortfall in our laboratory measurements. By this reasoning, the testbed has currently met our modeled fringe contrast expectations for COTS beam combiners and $632 \mathrm{~nm}$ light and we feel fairly confident that we understand the system well enough to move on to a monochromatic IR source.

After determining the cause and solution to several alignment limitations with the $632 \mathrm{~nm}$ source, we have now converted to a 1.15/3.39 micron source and have upgraded several optics. In particular, we have replaced all silver fold mirrors with uncoated gold and replaced COTS visible light beamsplitters with (50/50) IR beamsplitters. Some nearterm goals include the integration of hollow single-mode fibers for wavefront cleanup and the addition of a phase tracking control system. We will also conduct a study of techniques for the vernier control of the beam amplitudes. Three possible methods have been proposed. The simplest would be varying final beam combiner angles to cause slight changes in intensity. This approach, however, will change pathlength and may have other deleterious effects due to ghosting. The addition of another Michelson interferometer to the science path or a knife-edge mechanism will also be investigated. 
Phase tracking will be performed with a novel ditherless quadrature phase detector and a voice coil or piezo actuator driven in closed loop using a digital signal processing system. Picomotor actuators set on four mirrors, four beamsplitters and the cat's-eye phase-delays will be controlled with control system software in order to continuously track and steady the fringe during measurements. Fringe contrast will be further improved by mounting hollow fibers in the detector space to clean the wavefront of aberrations.

\section{THE DITHERLESS QUADRATURE PHASE DETECTOR}

Currently, the project seeks to test the ability to achieve a fringe contrast that is a $10^{4}$ null of central starlight. To accomplish this, wavefront errors will have to be reduced and the white light fringe must be tracked. Typically, modern optical and infrared stellar interferometers measure visibilities and phases of astronomical objects by electromechanically dithering a delay line about the central fringe position thereby changing the relative pathlength light travels from each siderostat or light-gathering telescope. Measurements of power are then taken periodically as the fringe is slewed using a saw-tooth drive wave delivered to an actuator that controls physical delay. A simple phase detection algorithm is subsequently used to derive the phase from these measurements. This signed error signal, which the control system seeks to minimize in magnitude, is then conditioned into a drive signal. This drive signal is used to change the relative pathlength so that the fringe contrast - the visibility - is consistently measured. The use of this approach to sensing and control of fringe position requires considerable time per cycle resulting in a typically slow, low-bandwidth response. A more efficient possibility is to introduce additional phase shifts optically and make all

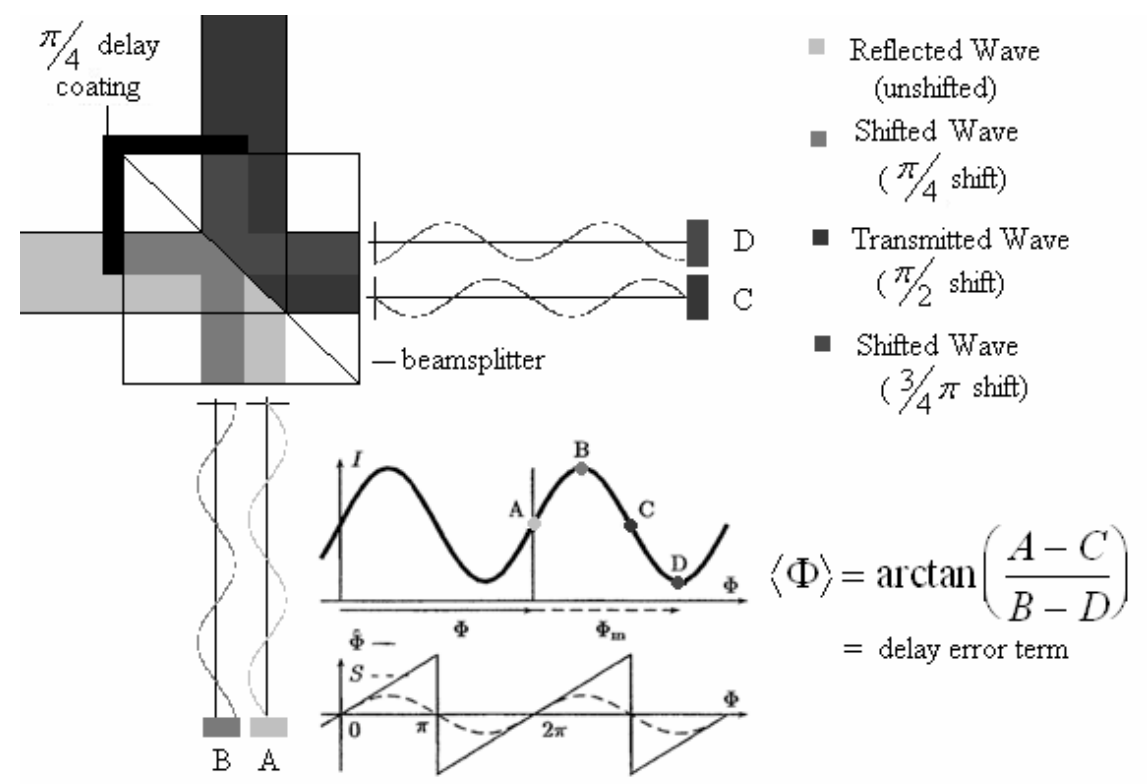

Fig. 6. The ditherless quadrature phase detector.

measurements simultaneously to produce the error signal. This could considerably stiffen the control system response and should result in greater fringe contrast, as there will be less movement of the fringe between measurements. This should, in turn, allow improvements to the overall sensitivity of instruments using this approach, as the integration time will not be fixed by the dither frequency of electromechanical transducers - typically the principle source of bandwidth limitation in any closed loop system. The Ditherless Quadrature Phase Detector (DQPD) is being developed contemporaneously with the FKSI testbed project and may be integrated in to it depending on the results of testing. The optical breadboard for the DQPD has been designed, and is being built in the Horizontal Flow Facility at GSFC on an optical table near the FKSI nulling testbed. 
The principle of the DQPD may be easily understood by examination of Fig. 6. This proof-of-concept design uses two beams, which are combined in a specially coated beam splitter. The coating, which delays half of the beam by $1 / 4 \pi$, is applied directly to the beam splitter, which is slightly wedged to reduce ghosting. The $1 / 2 \pi$ phase delay produced by transmission through the beam splitter results in the needed spread of measurements, which may be accomplished simultaneously using four detectors. The control system then rapidly calculates the required error signal as indicated in the figure. The optical breadboard is shown in Fig. 7.

Expected challenges for this phase sensor design are principally associated with our approach of using spatially separate portions of the wavefront to measure fringe power at different phases. If there are departures from ideal wavefront flatness or tilt, the error signal derived from the sensed phase will either have a systematic error term, which could be calibrated out, or will be noisy. We are now exploring designs that sample the same spatial patch of the wavefront at different polarizations or clean the wavefront and correct its tilt prior to sensing.

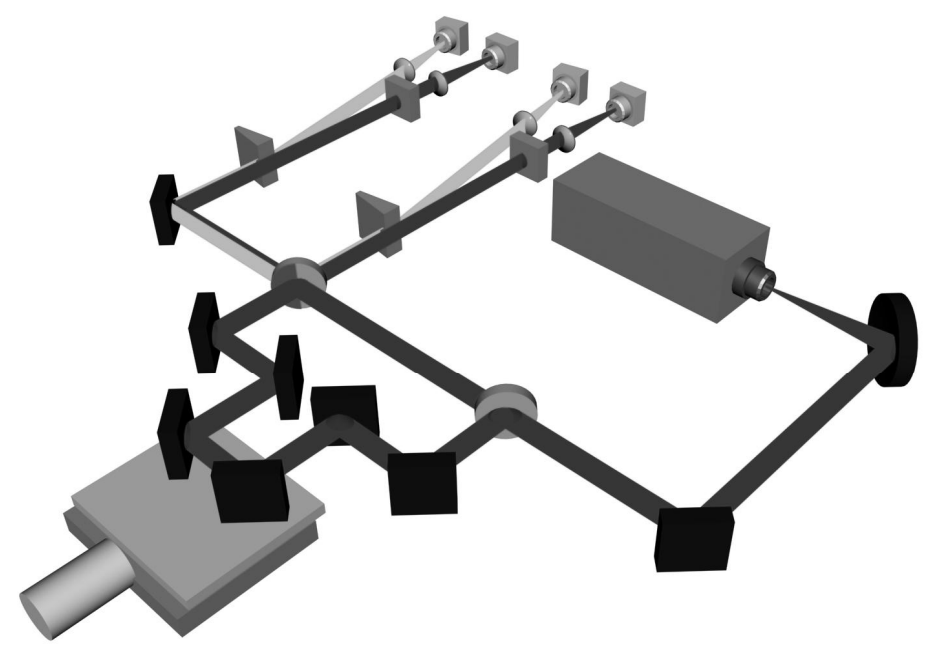

Fig. 7. The ditherless quadrature phase detector laboratory test setup.

\section{INSTRUMENT SYSTEM SIMULATION}

To assess the function of FKSI, the development team is using source and instrument simulators written in the IDL computer language. We use ZODIPIC, a software tool developed by Marc Kuchner to compute images of exozodiacal clouds [8]. These are built on observations of the solar system made by the Diffuse Infrared Background Experiment aboard the COBE satellite. FKSIM is a tool to simulate imaging with FKSI and is based on a simulator developed for the SIM mission [9]. Simulated images can be interactively computed by inputting the source model and various instrument parameters into the program's widget-based GUI like the number and spacing of apertures in the linear array, rotation angles, the wavelength and spectral resolution, integration time, etc. The tool also provides for deconvolving the raw images. The basic specifications and configuration of FKSI we have used for most of the images shown here are: Siderostat aperture size: $80 \mathrm{~cm}$., baseline: $20 \mathrm{~m}$., bandwidth: 1 micron at a center wavelength of 5 and 10 microns, respectively, and overall throughput: $10 \%$. For each image the array is rolled through $360^{\circ}$ in steps of $5^{\circ}$. At each roll-angle step, an image is captured in Fizeau mode. 

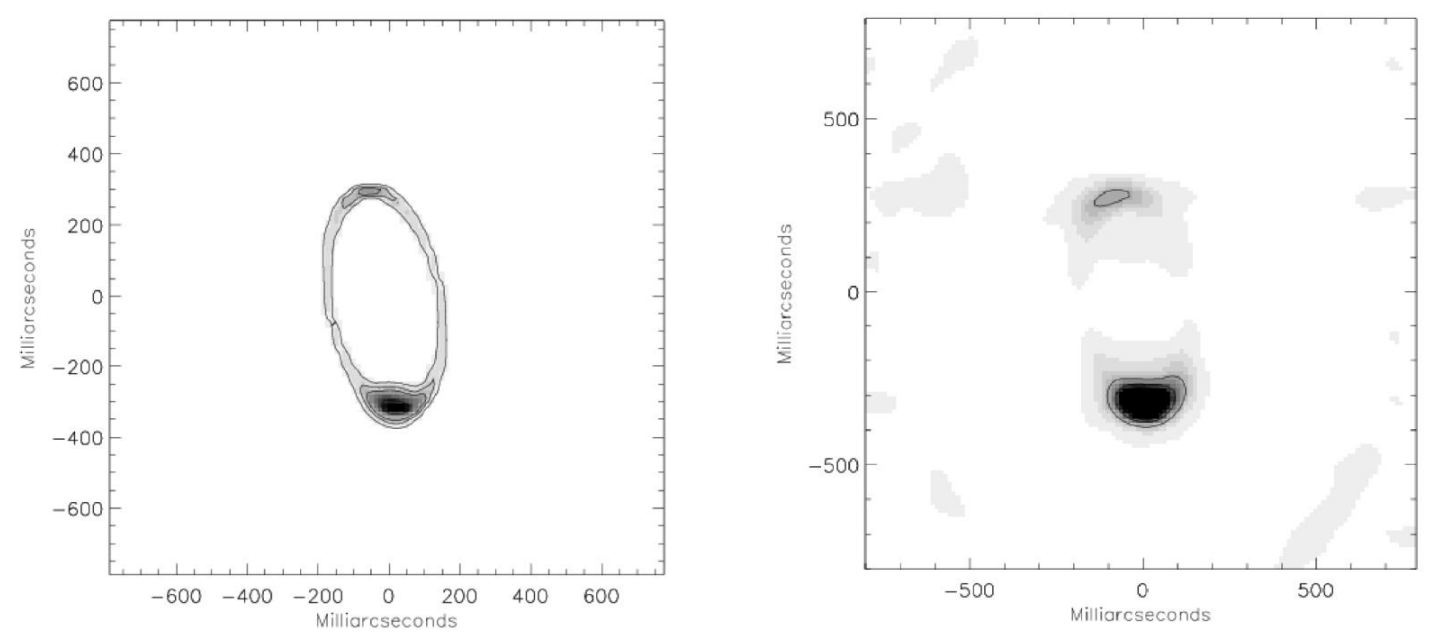

Fig. 8 Simulated source (left) and image at 5 microns - an Earth-like planet in a solar zodi about $\varepsilon$-Eridani with the smooth fan component and star flux suppressed to simulate the effect of nulling. The main features are recovered in spite of significant foreground local-zodiacal dust emission.
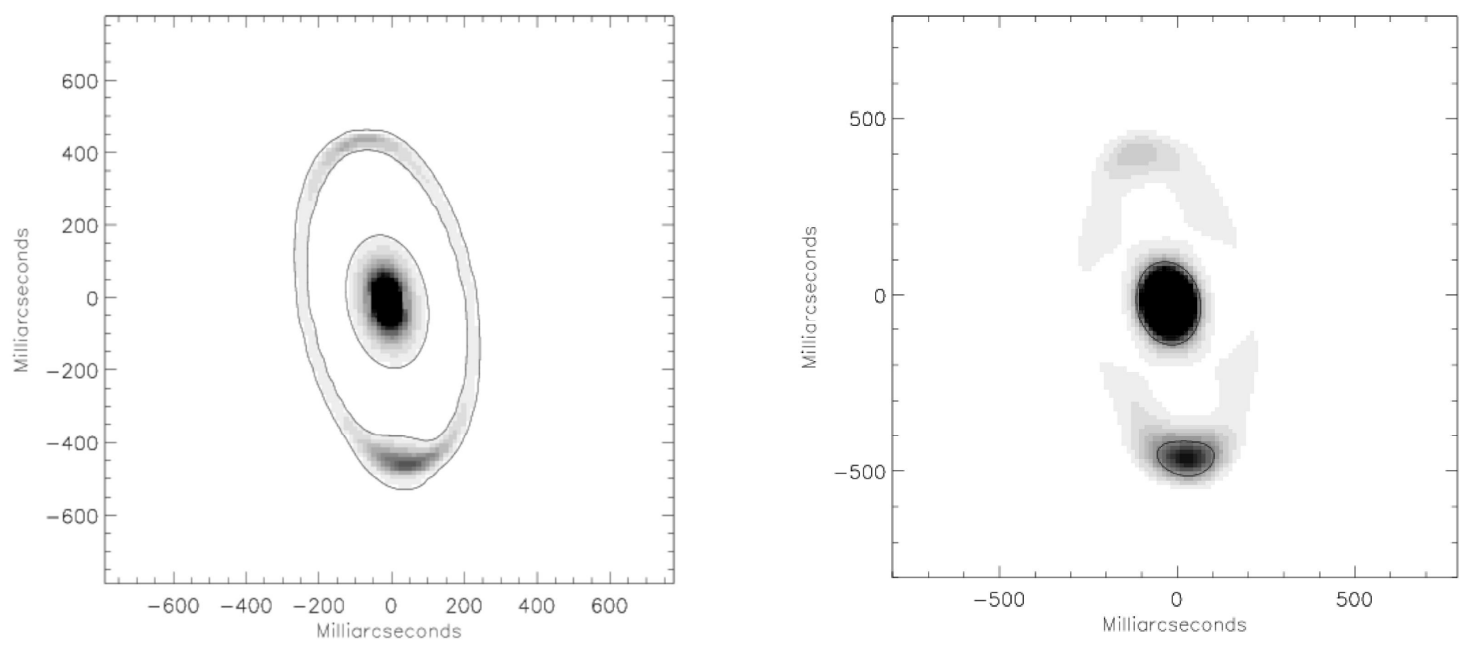

Fig. 9. Simulated source (left) and image at 10 microns for model source of a zodiacal cloud around $\varepsilon$-Eridani. The ring, which is at $1.5 \mathrm{AU}$, is enhanced by a factor of 1000 with star flux suppressed. The reconstruction includes thermal noise from mirrors at $40 \mathrm{~K}$ and emission from the local zodi.

These simulations provide a tool to understand the practical capabilities of the instrument at the design stage and the implications for the science goals. We find that at 5 microns, the thermal noise photons are not significant for the range of temperatures we include here. Photon noise and emission from the local zodi are significant, but do not prevent reasonable image quality. At 10 microns, thermal background and the local-zodi emission dominate and the simple deconvolution methods we use break down at telescope temperatures of $>80 \mathrm{~K}$ or so. These simulations start to show that a modest, medium baseline, connected-element interferometer like the FKSI is well capable of meeting the science goals of a TPF-precursor mission. The simulator is equipped to handle simple models for instrumental errors, and the next step will be to incorporate these effects into the simulations. Modeling the performance of the nuller is an important goal. 


\section{ACKNOWLEDGMENTS}

This work was supported in part by NASA Goddard Space Flight Center's Internal Research and Development (IRAD) and Director's Discretionary Fund (DDF) programs.

\section{REFERENCES}

[1] Danchi, W.C., Allen, R.J., Benford, D.J., Deming, D., Gezari, D.Y., Kuchner, M., Leisawitz, D.T., Linfield, R., Millan-Gabet, R., Monnier, J.D., Mumma, M., Mundy, L.G., Noecker, C., Rajagopal, J., Seager, S., Traub, W.A., 2003, Proc. "Towards Other Earths: DARWIN/TPF and the Search for Extrasolar Terrestrial Planets," Heidelberg, Germany, 22-25 April 2003 (ESA Publication SP-539)

[2] Danchi, W.C., Deming, D., Kuchner, M., \& Seager, S. 2003, ApJ, vol. 597, L57.

[3] Lopez, B., Schneider, J., Danchi, W.C., The Astrophysical Journal, 627:974-985, 2005 July 10

[4] Benford, D.J., Danchi, W.C., Allen, R.J., Deming, D., Gezari, D.Y., Kuchner, M., Leisawitz, D.T., Linfield, R., Millan-Gabet, R., Monnier, J.D., Mumma, M., Mundy, L.G., Noecker, C., Rajagopal, J., Seager, S., Traub, W.A., 2004, in the Proceedings of the October Maryland Conference.

[5] William C. Danchi, Ronald J. Allen, Dominic J. Benford, Drake Deming, Daniel Y. Gezari, Marc J. Kuchner, David T. Leisawitz, Roger P. Linfield, Rafael Millan-Gabet, John D. Monnier, Lee G. Mundy, Charley Noecker, Jayadev K. Rajagopal, L. J. Richardson, Stephen A. Rinehart, Sara Seager, Wesley A. Traub, Debra J. Wallace, Proc. SPIE Vol. 5491, p. 236-242, New Frontiers in Stellar Interferometry; Wesley A. Traub; Ed., SPIE, 2004

[6] T. Tupper Hyde, Kuo-Chia Liu, Carl Blaurock, Jeff Bolognese, Joseph M. Howard, William Danchi Proc. SPIE Vol. 5497, p. 553-564, Modeling and Systems Engineering for Astronomy; Simon C. Craig, Martin J. Cullum; Eds., SPIE, 2004

[7] Hyde, T.T., Liu, K-C., Blaurock, C. Bolognese, J., Howard, J.M., Danchi, W.C., Proc. SPIE Vol. 5491, New Frontiers in Stellar Interferometry; Wesley A. Traub; Ed., SPIE, 2004

[8] M. J. Kuchner 2002, IDL software program ZODIPIC (http://cfa-www.harvard.edu/ mkuchner).

[9] Rajagopal, J., Boeker, T., Allen, R.J., American Astronomical Society, 195th AAS Meeting, \#46.10; Bulletin of the American Astronomical Society, Vol. 31, p.144 